\title{
Golia di Beppe Fenoglio: lingue e Resistenza
}

\author{
Davide Torrent ${ }^{1}$
}

Ricevuto: 10 febbraio de 2019 / Modificato: 27 gennaio 2020 / Accettato: 3 marzo 2020

Riassunto. In questo contributo viene esaminato il racconto Golia di Beppe Fenoglio con l'obiettivo di individuare particolari scelte linguistiche e stilistiche finalizzate alla rappresentazione della comunicazione tra italiani e tedeschi. In particolare, si analizza l'uso del tedesco da parte degli italiani e viceversa. Grande risalto, inoltre, è dato ai procedimenti di semplificazione linguistica tipica dei discenti di una lingua sconosciuta. Dopo un confronto con altri testi di argomento simile, l'analisi si chiude con alcune proposte interpretative circa le scelte linguistiche determinate dalla concezione fenogliana della Resistenza.

Parole chiave: interlingua, comunicazione, resistenza, neorealismo, guerra civile.

\section{[en] Golia by Beppe Fenoglio: Languages and Resistance}

\begin{abstract}
This paper examines the tale Golia by Beppe Fenoglio, with the purpose of looking for peculiar representations of the communication between Italians and Germans. In particular, the study explores the German spoken by Italians and the Italian spoken by Germans. Particular attention is given to the typical habit of simplifying other languages. After a confrontation between the tale by Fenoglio and other tales and novels dealing with the same topic, the paper will address interpretative proposals of language choices.
\end{abstract}

Keywords: interlanguage, communication, resistance, neorealism, civil war.

Sommario: 1. Il racconto 2. Analisi formale 3. Conclusioni.

Come citare: Torrent, Davide (2020): «Golia di Beppe Fenoglio: lingue e Resistenza», Cuadernos de Filología Italiana, 27, pp. 255-274.

\section{Il racconto}

Golia viene pubblicato per la prima volta nel 1963 nel volume Un giorno di fuoco edito da Garzanti. Piera Tomasoni ipotizza che la presenza in questo volume, dall'orientamento prevalentemente langarolo-paesano, dei racconti Golia, Il padrone paga male e I premilitari risponda più che altro «all'economia editoriale di composizione del volume» (Fenoglio 1978: 633)². Dante Isella ci informa, nella scheda critica

\footnotetext{
1 Università degli studi di Torino. Dipartimento di Studi Umanistici, via sant'Ottavio, 20, Torino Email: torrent.davide@gmail.com

2 Si fa qui riferimento all'apparato critico a cura di Piera Tomasoni.
} 
riferita a questo racconto, che non se ne conserva «nessun manoscritto neppure frammentario, né menzioni di alcuna sorta» (Fenoglio 1992: 1658). La storia redazionale può essere ricostruita in base ai prestiti e ai contatti tematici con altri racconti e romanzi fenogliani, secondo quello che Maria Corti (1980: 10) chiama «processo di espansione della scrittura da un testo all'altro per cui ogni testo è insieme chiuso e aperto». C'è «un fondo tematico costante» (Corti 1970: 48) che si ripresenta frequentemente in testi dello stesso periodo. Per il racconto qui preso in esame si considerino l'immagine del partigiano appeso al chiodo da macellaio, che ritorna anche in Il padrone paga male (Fenoglio 1992: 1328)³, e quella di Polo che intende lavarsi nel sangue nemico, che ricorre in Una questione privata (Fenoglio 1992: 1055) e nella prima stesura del Partigiano Johnny (Fenoglio 1992: 526). Un ulteriore contatto col Partigiano è la descrizione dell'ufficiale vestito con una divisa inglese, confrontabile con la descrizione di Robin nel racconto (Fenoglio 1992: 588-589). I punti di contatto tra le opere di Fenoglio dimostrano che «alcuni materiali [...] si sdoppiano, si triplicano, danno origine a episodi imparentati, fratelli gemelli o fratelli cugini che poi vivono in diverse opere» (Corti 1970: 48). Proprio sulla base di tali elementi Isella propone una datazione tarda (Fenoglio 1992: 1658), sebbene il contatto con la prima stesura del Partigiano farebbe propendere per una meno tarda.

Sul piano tematico e contenutistico, Golia illustra i rapporti tra gli schieramenti impegnati nella guerra civile ma «non rispetta le regole sanzionate dal genere» (Guthmüller 2011: 146). I motivi che allontanano questo racconto dalle regole del genere sono vari, il primo dei quali è la commistione di elementi propri del racconto langarolo e di altri propri della narrativa partigiana (Guthmüller 2011: 146). L'elemento più inusuale è però il ruolo di protagonista ricoperto dal soldato tedesco prigioniero dei partigiani, indicato fin dall'inizio come «gigante» (Fenoglio 1978: 549) ${ }^{4}$. Il titolo richiama ovviamente l'episodio biblico della lotta tra il gigante Golia e il giovane Davide, nella quale si può scorgere l'immagine della guerra partigiana tra «l'empio, spaventoso Golia, potente per armi» (cioè l'armata tedesca) e «un Davide male armato, il quale però combatte per la giusta causa e a cui infine arride la vittoria» (Guthmüller 2011: 146-147). I personaggi del racconto sono numerosi, anche se quelli che svolgono un ruolo fondamentale per la progressione della trama sono in numero ridotto. Ovviamente il ruolo di protagonista è occupato dal prigioniero tedesco Fritz, al quale si contrappone il giovane partigiano Carnera. Tra gli altri partigiani, quelli portatori di un ruolo attivo sono Sandor, Polo e Ivan. Per quanto riguarda gli abitanti del paese si può riconoscere un ruolo rilevante nella storia soltanto al signor Ilario, padre della sposa di cui si narra in un episodio del racconto.

Il racconto narra della cattura di un soldato tedesco da parte di una compagnia di partigiani. Al suo arrivo il tedesco intimorisce la popolazione raccolta per osservarne l'ingresso in paese: «La gente fremette e serrò gomito a gomito quando su di essa, in curva, passò lo sguardo di lui» (GF: 550). Il suo sguardo però non è feroce, il prigioniero «che nell'aspetto corrisponde completamente al cliché del tedesco - è grande di statura, è biondo, ha occhi celesti, pelle bianca, è insensibile al freddo - è tutt'altro che un selvaggio bellicoso Golia») (Guthmüller 2011: 147). Il tedesco diventa un'attrazione per gli abitanti, sorpresi dalla novità della presenza di un soldato

Si fa qui riferimento all'apparato critico a cura di Dante Isella.

4 Da qui in avanti, e fino a nuova indicazione, le citazioni tratte da Fenoglio (1978: 549-574, vol. I) sono indicate dall'acronimo GF. 
dell'esercito occupante nel paese. L'interesse suscitato dal «tedesco meno tedesco che ci sia» (GF: 554) rappresenta uno dei temi fondamentali del racconto. Il suo essere profondamente diverso dall'immagine comune dei soldati tedeschi rappresenta l'espediente narrativo per la descrizione delle relazioni che si generano tra il prigioniero, la gente del paese, i partigiani e soprattutto Carnera, che incarna il Davide del racconto. La straordinarietà della prigionia di un soldato tedesco è rilevata ogni volta che entra in scena. I paesani meravigliati osservano un prigioniero che spiega «il mistero dell'otto settembre» (GF: 550), membro di quell'esercito tedesco da cui dipende l'esito della guerra ${ }^{5}$, sebbene già dai primi giorni «non lo considerassero più un nemico» (GF: 553). L'evento paesano più rilevante è un matrimonio a cui Fritz viene invitato quasi come ospite d'onore. Gli invitati sostengono più volte che il tedesco «è dei nostri» (GF: 562; 563; 566), eliminando la distanza che li separa dal prigioniero. Il rapporto che nel corso del racconto si instaura non solo con gli abitanti del paese ma anche con i partigiani si rivela dunque molto diverso rispetto al tradizionale rapporto tra nemici. Proprio su questo tema si sviluppa un'accesa discussione tra i partigiani, tra i quali sorge il dubbio che il prigioniero sia trattato «scandalosamente bene» (GF: 553). Proprio in questa discussione si vede il tema fondamentale trattato da questo racconto, cioè una particolare descrizione della guerra civile. Soltanto una minoranza dei partigiani riconosce in Fritz un nemico, dal momento che quasi tutti lo considerano «un tipo domestico» (GF: 554). Le parole di Ivan, il partigiano che chiede la fucilazione del prigioniero come vendetta per i compagni caduti, illustrano chiaramente l'idea di guerra diffusa nelle file della Resistenza:

Ma che gente siamo noi italiani? Siamo in una guerra in cui si può far del male a tutti, si deve far del male a tutti, e noi ce lo facciamo soltanto tra noi. Cos'è questo? Vigliaccheria, cretina bontà, forse giustizia? Io non lo so. So solo che se noi di qua pigliamo un tedesco, invece di ammazzarlo finiamo per tenerlo come uno dei nostri. I fascisti di là, se beccano un inglese o un americano, qualche sfregio certo gli faranno, ma ammazzarlo non lo ammazzano. Ma se invece ci pigliamo tra noi, niente ti salva più, e se cerchiamo di spiegare che siamo fratelli ci ridiamo in faccia. E così, quando la guerra finirà, ci sarà, mettiamo, degli inglesi che tornano dalle loro madri e dicono: «M'hanno preso i fascisti italiani ma m'hanno lasciata salva la vita», e dei tedeschi torneranno a casa e diranno la stessa cosa dei partigiani italiani. Ma alle madri italiane, alle nostre, che cosa si dirà? (GF: 555-556)

Il prigioniero non viene fucilato $\mathrm{e}$, nonostante le perplessità di alcuni partigiani, conserva il suo status privilegiato. Neppure in seguito alla morte di Tarzan ad opera dei fascisti, quando «il tedesco dovette tremare per la sua vita» (GF: 557), si pensò di vendicare la morte del caduto con l'esecuzione di Fritz. Mentre Polo finge di lavarsi le mani in un catino immaginario colmo del sangue dei nemici (GF: 559), Fritz si crede ormai prossimo alla morte ma viene completamente trascurato. Tale episodio non rappresenta una «digressione dalla tematica centrale» ma «rende invece ancor

«Voi, poveri ragazzi, lontani dalle vostre case, fate tutto quello che potete, ma non potete far altro che star quassù a difendervi, con poco o niente, e a patire. Voi lo sapete solo quanto me quando la guerra finisce. Fritz invece lo sa, lui è dell'esercito tedesco, e tutto dipende dall'esercito tedesco» (GF: 565). 
più palese che i partigiani non riescano più a vedere in lui il nemico» (Guthmüller 2011: 147). Le parole di Ivan sopra citate permettono di cogliere l'idea di Resistenza di Fenoglio. Come riconoscono gli stessi partigiani, il nemico principale non è l'occupante tedesco ma l'esercito fascista. La guerra si caratterizza principalmente come una guerra civile «dove l'odio per il nemico italiano è superiore a quello provato verso il nemico straniero» (Bufano 1999: 92) e lo stesso Fritz rileva questa idea propria dei partigiani che lo tengono prigioniero: «la cosa stava tutta tra italiani» (GF: 560).

Il partigiano che più prende a cuore la causa del tradimento dei compagni caduti è Carnera, ironicamente chiamato col nome del noto pugile. Infatti, Carnera, di soli quattordici anni, è il più piccolo tra i partigiani. Rappresenta l'antagonista di Fritz nel racconto e la descrizione dei due personaggi li differenzia molto dettagliatamente anche dal punto di vista caratteriale. Il tedesco è «inoffensivo e bonario» (Guthmüller 2011: 152), mentre il giovane partigiano viene descritto come «aggressivo e fastidioso» (Guthmüller 2011: 152). Lo scontro tra i due personaggi si sviluppa nel corso di tutto il racconto ma fin dal loro primo incontro si intuisce l'esito che avrà la vicenda. All'ingresso del tedesco in paese, Carnera gli salta al petto per strappargli una medaglia, mentre «il colosso si portò una mano al petto come se lì fosse stato ferito» (GF: 550). La violenza del primo incontro tra i due si ripete alla fine del racconto, quando, prima di essere ucciso, il tedesco «si tastava tutto il petto, come per misurarlo per sé e per Carnera» (GF: 574). Sarà infatti il più piccolo tra i partigiani a uccidere Fritz. Egli rappresenta «il giovane partigiano fanatico che vede nel nemico solo il nemico» (Guthmüller 2011: 153). Il suo fanatismo è dovuto anche al poco rispetto che gli altri partigiani dimostrano nei suoi confronti poiché troppo giovane, il che lo spinge a mostrarsi come in grado di agire, nella sua prospettiva, da vero partigiano privo di pietà nei confronti del nemico e pronto a uccidere. Proprio dal carattere del più piccolo tra i partigiani viene generato il sospetto che «tutto il fanatismo partigiano di Carnera non nasca da un impegno per la causa comune ma che si tratti piuttosto di compensare sensi di inferiorità e inappagati desideri di lode» (Guthmüller 2011: 153).

L'epilogo della vicenda si ha quando a Carnera viene impedito di unirsi ai suoi compagni per raggiungere gli ufficiali inglesi, per «il pericolo che si formino il concetto che noi partigiani non siamo una cosa seria» (GF: 569). Dopo che Carnera propone a Fritz un giro intorno al paese, i due si dirigono verso un bosco e il partigiano si spaventa della situazione: «E se questo tedesco si convince che io sono piccolo, un ragazzino qualunque? [...] Ma la mia pistola sparerà? Non 1'ho mai provata» (GF: 572). Il diverbio che si accende tra i due porta il tedesco a ricordare per tre volte a Carnera la sua inadeguatezza come partigiano: «Tu piccolo. Non essere capace di uccidere me» (GF: 574). L'uccisione di Fritz non si rivela dunque un'eroica impresa partigiana ma «non è altro che un arbitrario atto di violenza, compiuto da un ragazzo fanatico che non riesce a dominarsi e a sopportare di non essere preso sul serio come partigiano» (Guthmüller 2011: 155). L'azione di Carnera non si colloca dunque nell'interesse collettivo né in una posizione rilevante per la lotta partigiana. L'esecuzione del tedesco, perché tale è l'uccisione di Fritz, non rappresenta una scelta di giustizia come reclamato dal partigiano Ivan per vendicare l'uccisione di molti compagni caduti. La scelta dell'uccisione del tedesco ricade interamente sul giovane partigiano che agisce per sé solo.

Con la consapevolezza che «era ormai finita 1'epoca della rappresentazione a senso unico della guerra» (De Nicola 1996: 50), Fenoglio costruisce un racconto in 
grado di mettere in dubbio la rigida schematicità della rappresentazione della guerra civile. L'operazione fenogliana risulta dunque non una dissacrazione del mito resistenziale ma semmai una «analisi critica (e dal basso) della Resistenza» (Bigazzi 1983: 17). Pur riconoscendo senza ombra di dubbio che a livello storico-politico la ragione risiede nello schieramento partigiano, «questo non comporta però necessariamente che il bene e il male, gli orrori della guerra, le inutili violenze, la pietà e l'umanità siano distribuiti secondo criteri politici e nazionali» (Guthmüller 2011: 155). L'oggetto con cui Fenoglio si confronta non è soltanto quello della lotta tra partigiani e nazifascisti. Il suo obiettivo è quello di «rappresentare il soggetto nel suo rapporto con la scelta» (Alfano 2014: 749), in questo caso rivoluzionando gli schemi della letteratura resistenziale, ponendo al centro del racconto un bonario e non pericoloso soldato tedesco contrapposto a un partigiano giovane e crudele che compie la scelta di uccidere l'avversario con motivazioni insolite rispetto ai canonici racconti partigiani. Si può riconoscere proprio in questa novità tematica un elemento di allontanamento di Fenoglio dalla letteratura resistenziale e dai suoi schemi. Il racconto propone invece una visione problematica sia delle motivazioni della lotta partigiana sia del confronto tra partigiani (buoni) e tedeschi (cattivi). L'operazione di Fenoglio risulta dunque più complessa di un semplice parallelo tra la guerra partigiana a e l'episodio biblico in quanto la polarità tra bene e male viene meno e con essa inizia ad essere messa in discussione l'immagine dei «"partigiani assoluti”, perfetti, fulgidi e motivati» (Sipione 2011: 90) che così spesso ricorrono nella letteratura partigiana. L'intento è quello di «scartare la via consueta del genere (tesa a celebrare i valori) per insistere sull'asprezza della lotta» (Bigazzi 1983: 15) e il titolo mette in risalto la straniante rappresentazione di una lotta tra Davide e Golia ribaltata in ogni suo aspetto. Emerge dal racconto, per questi motivi, «la ricerca di un rapporto col reale meno programmaticamente ottimistico o incline alla retorica resistenziale e populistica, ma, proprio per questo, più crudo e rigoroso nella rappresentazione» (Chicco Vitizzai 1977: 42).

\section{Analisi formale}

Il racconto Golia è narrato in terza persona. Il narratore risulta interno alla narrazione. Nella maggior parte del narratum è riconoscibile l'esatta corrispondenza tra il punto di vista degli abitanti del paese e quello del narratore. Come si è già accennato, Golia presenta elementi propri dei racconti partigiani e altri propri di quelli langhigiani. Vale per questo racconto ciò che afferma Maria Antonietta Grignani a proposito dei racconti langhigiani: «Anche le parti narrative sono protese a cogliere dall'interno la visione del mondo che inerisce ai protagonisti» (Grignani 1981: 85). In più punti del racconto sono inserite frasi che riportano in discorso indiretto libero le opinioni degli abitanti del paese, tra le quali si confonde quella del narratore: «Gli trovarono un'aria grave come non mai, come se sentisse il peso di una nuova e più alta responsabilità» (GF: 550), «Il fatto però non danneggiò Fritz in paese, dove la cosa si riseppe, perché era chiaro che la colpa era tutta di lei» (GF: 552-553), «erano infinitamente più stranieri i due prigionieri inglesi, Tom e Victory, che l'otto settembre erano evasi da un campo di concentramento ed erano stati qualche mese con Sandor finché in uno sbandamento del ' 44 erano andati a perdersi chissà dove» (GF: 553), «e adesso chissà che andava a capitare» (GF: 565). Allo stesso procedimento 
di rappresentazione del carattere collettivo va ascritta la fitta presenza dello stile impersonale - «si vide» (GF: 550), «si sentiva» (GF: 556), «si sentì (GF: 558), «tutto fu visto» (GF: 558), «si sbirciò» (GF: 565) - e di deittici temporali - «l'ultima volta», «l'ultima volta e la penultima», «stavolta», «ora», «già», «adesso» - e spaziali - «là dentro», «là dove», «da lassù», «lì»-. La voce narrante si adatta anche al punto di vista di Fritz, reso attraverso il discorso indiretto libero: «Conosceva la natura sottostante: una scarpata da potersi far rotoloni in un niente, quindi una raggera di rittani [...]. Ma poi?», «dovevano non vederlo, non ricordarsi di lui, non indicarselo l'un l'altro» (GF: 559).

Il tempo della storia e il tempo della narrazione tendono a non coincidere. Il racconto si configura come la successione di una serie di scene alle quali viene concesso uno spazio variabile. Si possono identificare otto episodi di lunghezza variabile: l'arrivo in paese del prigioniero (GF: 549-551), l'incontro con gli abitanti del paese (GF: 551-553), la discussione del gruppo partigiano (GF: 553-557), la notizia della morte di Tarzan (GF: 557-560), il matrimonio (GF: 560-566), la nevicata (GF: 566-567), l'arrivo dell'ufficiale (GF: 567-571) e il conseguente scontro tra Fritz e Carnera (GF: 571-574). Poco spazio è lasciato alle parti descrittive, quasi assenti. La maggior parte dello spazio è occupato dai dialoghi, che appaiono per la maggior parte raccolti a gruppi in sezioni interamente dialogate formate da discorsi diretti, anche se nel narratum si riscontrano casi di discorso indiretto dei quali si è già parlato.

In questo senso si può intendere la struttura del racconto come assimilabile a un modello «centrato sulla martellata forma dialogica, violentemente contrastiva, con bruschi salti tra un momento e l'altro della narrazione» (Soletti 1983: 156). Tale struttura narrativa, nella quale «il dialogato risulta la macrostruttura formale portante» (Corti 1978: 79) sembrerebbe collocare il racconto all'interno della narrativa comunemente raccolta sotto l'etichetta di neorealista. Risultano però necessarie, a questo proposito, due osservazioni. È fondamentale innanzitutto considerare il fatto che, accogliendo le ipotesi di datazione discusse all'inizio del presente lavoro, Golia andrebbe collocato al di fuori del periodo al quale viene solitamente ricondotta la fase neorealista che secondo Francesco De Nicola (1996: 48) non andò oltre la pubblicazione di Metello di Pratolini nel 1955. In secondo luogo, occorre riconoscere «l'inadeguatezza del termine "neorealismo"» (Milanini 1980: 7) per racchiudere la varietà di autori e di tendenze osservabili nel dopoguerra. Con la consapevolezza che «ogni tentativo di definire oggi il Neorealismo finisce $[. .$.$] per evidenziarne una presunta eterogeneità interna» (Baldini$ 2013: 109), tale categoria sarà utilizzata come termine di paragone per l'analisi di Golia unicamente riconoscendone la natura problematica e per nulla definitiva. Un'ulteriore specificazione appare necessaria prima di analizzare il racconto. La suddivisione in tre fasi del fenomeno neorealista proposta da Romano Luperini permette di riconoscere con più chiarezza il termine di paragone funzionale alla comprensione della novità rappresentata da Golia. Se ovviamente il «realismo politico» (Luperini 2001: 132) degli anni '30 appare lontano dal lavoro fenogliano, la stessa lontananza può essere riscontrata anche rispetto alle due fasi successive indicate (Luperini 2001: 132) come «realismo mitico-simbolico» (1940-1948) e «realismo socialista» (1949-1955), come si cercherà di dimostrare nel presente lavoro. La prospettiva di Fenoglio, estranea «agli intenti marcatamente ideologici e politici» (Luperini 2001: 127) che secondo Luperini accomunano le tre fasi, si colloca al di fuori di qualsiasi definizione di neorealismo formulata dalla critica e mette in discussione gli assunti ideologici che fino a quel momento rappresentavano la principale chiave di lettura della lotta resistenziale. 
L'analisi linguistica del racconto si articolerà distinguendo le occorrenze dei vari fenomeni nelle parti dialogate e in quelle narrative. Ossia, considerata la centralità accordata al dialogato nel racconto, proprio sul dato caratterizzante i dialoghi ci si concentrerà sull'uso di un italiano di apprendimento da parte del prigioniero tedesco e sui riverberi che questo provoca nei madrelingua italiani. Non solo la rappresentazione di questi fenomeni è così aderente al reale da permettere di analizzarli con un manuale di sociolinguistica alla mano, ma si tratta anche di un tema non così frequente nella letteratura resistenziale.

Una delle caratteristiche principali del racconto è l'elevata frequenza di tratti linguistici significativi che collocano l'ipotetica lingua del tedesco in un contesto di estrema semplificazione, caratteristica propria della lingua di un discente. Prevale l'utilizzo di verbi al modo infinito - «Voi aggiustare e riportare» (GF: 551), «Tu cercare me?» (GF: 560), «Sapere valzer delle bimbe brune?» (GF: 562), «io non sapere altre» (GF: 562), «sapere tante altre cose» (GF: 562), «io dire tutto quello che io sapere» (GF: 565), ecc - o al participio - «stivale rotto in una parte» (551), «quando preso me, agito da vero soldato» (GF: 560) - e di conseguenza si può notare che i casi in cui i verbi sono coniugati sono in numero molto ridotto - «Sandor ti manda?» (GF: 560), «Buono era Tarzan» (GF: 560), «anche se sono da lungo tempo» (GF: 565), «come dice il padrone» (GF: 565), «voi volete sapere» (GF: 565), «se guerra finisce, tutto il mondo è felice» (GF: 565-566), «io amo boschi» (GF: 572) -, concentrati per la maggior parte (quattro occorrenze sulle sette totali) nel dialogo che il tedesco intrattiene con il signor Ilario. Gli articoli sono quasi completamente assenti, dal momento che ricorrono soltanto sette volte nella totalità delle frasi pronunciate da Fritz - «una parte» (GF: 551), «il padrone» (GF: 565), «almeno un nemico» (GF: 565), «il tempo» (GF: 565), «una parola» (GF: 565), «il mondo» (GF: 566), «il partigiano» (GF: 573) -, anche in questo caso con cinque casi concentrati nel discorso con il signor Ilario. A questo aspetto è necessario aggiungere l'utilizzo molto semplificato o l'assenza delle preposizioni: si alternano preposizioni semplici e articolate in contesti simili («dai miei camerati» / «da mio esercito tedesco»), la preposizione «a» viene utilizzata come unico introduttore del complemento di moto a luogo - «andiamo a bosco» (GF: 572) -, le preposizioni utilizzate per l'introduzione di verbi sono omesse - «capace fare affari» (GF: 571) -.

La scarsa conoscenza linguistica del tedesco agisce anche sull'organizzazione della sintassi che mostra alcune caratteristiche peculiari, come la presenza diffusa di sintassi nominale (Grignani 1978: 320), realizzata soprattutto attraverso processi di ellissi del verbo - «Belli bambini» (551), «Kartoffel, patate, ja» (563), «Germania non più forte da vincere guerra» (GF: 565), «Tutti gli altri non adatti, e voi italiani meno di tutti» (GF: 565), «capace, sì (GF: 567), «popolo molto serio, popolo molto capace fare affari» (GF: 571), «No, niente lontano» (GF: 572), «io non buono soldato tedesco, ma anche tu non buono partigiano» (GF: 573), «tu piccolo» (GF: 574) -, attraverso la preferenza per la coordinazione per asindeto - «soldati tedeschi essere tutti eroi, essere molto pochi quelli come Fritz» (GF: 565), «soldato tedesco non morire mai solo, portare con sé almeno un nemico» (GF: 565), «Tu essere piccolo, dovere stare a scuola» (GF: 573) - o attraverso congiunzioni esclusivamente copulative - quattro occorrenze della congiunzione «e», come ad esempio «voi essere italiani, e solo tedeschi adatti a capire tedeschi» (GF: 565) - e avversative - sei occorrenze di «ma», come ad esempio «No, io non sapere altre canze, ma sapere tante altre cose buone per festa...» (GF: 564), «Ora molti soldati tedeschi morire, molti molti, ma morire anche molti suoi nemici» (GF: 565) -. 
La semplificazione linguistica messa in atto dal tedesco si può osservare anche nella scarsità di proposizioni subordinate, limitate a una temporale - «quando preso me» (GF: 560) -, una causale - «perché soldato tedesco non morire mai solo» (GF: 565) -, una concessiva («anche se sono da lungo tempo separato dai miei camerati» (GF: 565)), una comparativa - «come dice il padrone della casa» (GF: 565) -, tre relative - «che stare a bere vino» (GF: 565 ) -, due consecutive - «da farla andare lunga» (GF: 565) - e da un periodo ipotetico della realtà - «se guerra finisce, tutto il mondo è felice» (GF: 565-566) -. Tali fenomeni dimostrano il peculiare interesse di Fenoglio nella rappresentazione della particolare lingua utilizzata per comunicare da un individuo che conosce poco l'italiano. L'insieme dei tratti che caratterizzano la lingua di Fritz sembra convergere sulle descrizioni di quella che è stata definita interlanguage, categoria sviluppata da Selinker (1972), cioè una lingua originata dalla sovrapposizione in un parlante monolingua di una lingua seconda non perfettamente acquisita (Berruto 2003: 163-168; Chini 2005: 80-87, 94-110). Con la dovuta accortezza, in quanto si tratta di un concetto sviluppato decenni dopo la stesura di Golia, si può riconoscere l'utilità di una simile categoria nell'analisi critica di un racconto che intende mettere in scena l'atto pratico della comunicazione tra personaggi parlanti lingue differenti che, sebbene contrapposti militarmente e politicamente, intendono dare avvio a un dialogo che risulterebbe altrimenti impossibile.

Alcuni di questi elementi sono riscontrabili anche in altre opere resistenziali. In particolare, le stesse caratteristiche riscontrate nel racconto di Fenoglio si possono osservare nel racconto Estate che mai dimenticheremo, pubblicato da Marcello Venturi sul Politecnico nel 1945 (Pedullà 2005). Il racconto, pubblicato a caldo subito dopo la guerra, narra un episodio avvenuto durante l'estate del 1944. Un soldato tedesco, fermatosi presso la casa di un contadino per riparare una ruota forata, parla con l'uomo, mettendo in atto tutte le principali caratteristiche già elencate dell'interlingua propria di un parlante tedesco: la sintassi nominale-«Questo, tuo bambino?», «E quella, tua moglie?», «Presto io Germania, - disse il soldato. - Tutto kaputt e io Germania», «Guerra niente buono», «Gomma kaputt. Ora niente kaputt», «Ma molto caldo. [...] E molta fame», «Italiani sempre nulla» (Pedullà 2005: 305-307), ecc. -, i verbi coniugati prevalentemente all'infinito - «Io piacere molto bambino» «Io avere casa molti bambini» (Pedullà 2005: 305), «Tornare Germania, io» (Pedullà 2005: 306), «Aspettare notte, - disse. - Ora soffocare» (Pedullà 2005: 309), ecc. - o al participio - «Presto tutto finito» (Pedullà 2005: 306) -, l'assenza quasi totale di proposizioni subordinate ad esclusione di una temporale - «Quando sole dormire» (Pedullà 2005: 307) - e di una causale - «Io stare qui perché caldo con la macchina» (Pedullà 2005: 309) -, la concordanza straniante di verbo e soggetto - «A me piace $\mathrm{i}$ bambini» (Pedullà 2005: 305) -, l'assenza delle preposizioni - «Io avere casa molti bambini», «Tronare Germania, io» (Pedullà 2005: 305, 306); ecc. - .

Anche in Banditi di Pietro Chiodi (1946 [2015]) sono frequenti i dialoghi in cui sono presenti dei soldati tedeschi con scarsa conoscenza della nostra lingua. Si possono trovare anche in questo caso gli stessi fenomeni analizzati in precedenza: la diffusa presenza di verbi all'infinito - «Se vincere loro fare caput a noi. Noi oggi fare caput a loro» (Chiodi 1946 [2015: 27]), «Non potere avvicinarsi ai condannati», «No, non potere. Se poi il prete rispondere alle domande del condannato quale punizione dare al prete?» (Chiodi 1946 [2015: 153]), «Questo essere uomo» (Chiodi 1946 [2015: 154]), «Sì mandare a dire a dire alla famiglia quanto ha detto il condannato senza mettere né dove, né come, né quando» (Chiodi 1946 [2015: 155]), «Do- 
mani dopo mezzogiorno fare quello che volete, noi non interessare più», «Noi avere fretta», «Se loro non aver pensato alla famiglia all'ultimo momento noi non potere fare niente. Scrivere solo a quelli ma senza indicazione del luogo» (Chiodi 1946 [2015: 156]) - e lo stile nominale -«Via, caput» (Chiodi 1946 [2015: 24]), «Brutto ordine, brutto comando» (Chiodi 1946 [2015: 156]) -.

Nella narrativa resistenziale, personaggi madrelingua tedeschi alle prese con 1'italiano si possono trovare anche in altre opere. Calvino nel Sentiero dei nidi di ragno rappresenta all'inizio del romanzo un tedesco, cliente della sorella del protagonista Pin. Le parole pronunciate dal tedesco - «niente sigarette avere» (Calvino 1947 [2011: 7]), «sorella non in casa?» (Calvino 1947 [2011]: 8) - si collocano perfettamente tra i fenomeni qui analizzati. Si può notare, infatti, che il verbo della prima frase è all'infinito, mentre la seconda frase presenta un'ellissi del verbo che la rende nominale. Entrambi questi fenomeni sono riconducibili alla semplificazione linguistica messa in atto da un parlante non italiano con scarsa conoscenza della nostra lingua. In Uomini e no, il romanzo di Vittorini «più prossimo ai canoni neorealisti» (Matt 2011: 74), si possono osservare inserti di parlato dei tedeschi. Anche in questo caso ricorrono alcuni elementi propri della rappresentazione della difficoltà linguistica dei non parlanti italiano, come la presenza dei verbi al modo infinito - «Contare. Contare con io» (Vittorini 1945 [1981: 67]), «Provare tu» (Vittorini 1945 [1981: $68]$ ) - e l'uso straniato del pronome personale «io». Per quanto si possa notare una maggiore frequenza di dialoghi di tedeschi alle prese con l'italiano nei testi di Venturi e di Chiodi, i fenomeni rilevati in tutti e quattro gli autori danno l'impressione di una rappresentazione schematica e semplificata, non realmente interessata alla realtà di queste varietà di apprendimento dell'italiano, a differenza di quanto avviene in Golia, dove i tratti introdotti sono più numerosi e variati.

Non solo questi elementi si trovano nella lingua di Fritz ma possono essere osservati anche nei discorsi pronunciati dagli italiani interagendo con il tedesco. Si può ipotizzare che tale procedura di semplificazione linguistica messa in atto da parlanti italiani rappresenti un tentativo di abbassamento stilistico volto a facilitare la comunicazione con il tedesco, messo in difficoltà dalla lingua. Tale procedimento può essere assunto come efficace rappresentazione della

teoria dell'accomodazione (o accomodamento o adeguamento), secondo cui i parlanti nell'interazione verbale tenderebbero, all'interno del repertorio a loro disposizione, a convergere in varie caratteristiche del comportamento verbale [...] verso il modo, reale o attribuito, in cui parla l'interlocutore, rendendo più simili $i$ rispettivi modi di parlare. (Berruto 2003: 74)

Si possono tuttavia riscontrare alcune differenze tra la semplificazione messa in atto da Fritz e quella degli italiani. Le forme flesse del verbo sono utilizzate più diffusamente - «Chi te l'ha insegnata, Fritz?» (GF: 562), «ne sai altre, Fritz?» (GF: 562), «Ma la gente non era cattiva, era buona» (GF: 564), «Perché non vuoi?» (GF: 567), «Andiamo a fare un giro intorno al paese» (GF: 571) -, mescolate senza apparente ordine alle forme all'infinito - «Io sapere aggiustare. Aggiustare e riportare» (GF: 551), «tu non andare a dormire?» (GF: 560), «anch'io essere stato soldato, [...] avere fatto tutta l'altra guerra» (GF: 563), ecc. - . A differenza di Fritz, gli italiani mostrano un maggior rigore nell'osservanza delle regole della loro lingua materna, della quale conservano gli articoli, ad esclusione di poche eccezioni - «essere alpino» 
(GF: 563), «mia figlia e suo uomo» (GF: 564) -, e utilizzano in maniera adeguata le preposizioni, salvo poche eccezioni - «ai tempi di mia gioventù» (GF: 563), «Perché proprio a bosco?» (GF: 572) -. Per quanto riguarda l'organizzazione sintattica del testo, si può osservare una minore semplificazione linguistica, che si realizza con la presenza estremamente ridotta dello stile nominale - «alpini, quei soldati italiani con una piuma sul cappello» (GF: 563), «Tanta fame in Austria, e tanto freddo» (GF: 564), «Ma mia figlia e quelli giovani come lei?» (GF: 564) -, la presenza di frasi generalmente brevi ma collegate da una maggiore varietà di congiunzioni copulative - «Noi siamo vecchi e frusti, e quando la morte viene, viene sempre all'ora giusta, e magari anche un po' tardi» (GF: 564) -, avversative - «Anche Sandor e tutti gli altri non sono buoni partigiani, ma ci mettiamo poco, sai, a diventare buoni partigiani» (GF: 573) -, conclusive - «mia figlia può perdere il suo uomo, e allora tutto il bene che io le ho fatto non le servirebbe più a niente» (GF: 564) -. A livello ipotattico si può riscontrare la maggiore frequenza di proposizioni subordinate molto diversificate: oggettive - «Voi lo sapete solo quanto me quando la guerra finisce» (GF: $565)$-, temporali - «quando ci hanno portato prigionieri in Austria, là ci davano da mangiare patate» (GF: 564) -, causali - «perché anch'io essere soldato, ai tempi di mia gioventù avere fatto tutta l'altra guerra» (GF: 563) -, finali - «Tutta la vita ho lavorato per procurar del bene a mia figlia» (GF: 564) -, relative - «tutto il bene che io le ho fatto non le servirebbe più a niente» (GF: 564) - e di periodi ipotetici -«se andare ancora lunga, noi tutti moriamo di crepacuore» (GF: 564) -.

A queste caratteristiche, limitate ai dialoghi tra italiani e tedeschi se ne aggiungono altre, espressioni della forzatura dell'ordine naturale della frase che Elisabetta Soletti (1987: 62) identifica nella lingua fenogliana, diffuse in tutti i discorsi pronunciati dagli italiani. Si può notare una fitta presenza di fenomeni dell'oralità quali frasi scisse e pseudoscisse - «questo è lo scandaloso che dico io» (GF: 554), «son proprio gli estranei, gli innocenti, che il più delle volte ci lasciano la pelle» (GF: 561), «siamo noi che dobbiamo ringraziare voi» (GF: 566), «il tuo uomo è di quelli che se ne stanno a casa» (GF: 561), «c'è il cuciniere che prima o poi ci mette a lavorare» (GF: 571) -, frasi segmentate - «non lo vedi che è il tedesco meno tedesco che ci sia?» (GF: 554), «te lo dico io quel che sei venuto a far tu nei partigiani» (GF: 556), «io che me ne faccio poi della vita?» (GF: 561), «noi prigionieri lo vedevamo da noi che la gente non avere pane nemmeno per lei» (GF: 564), «voi lo sapete solo quanto me quando la guerra finisce» (GF: 565), «quel piccolo di prima basta lui a tenerlo d'occhio» (GF: 570), «queste cose lasciamole fare ai Muti» (GF: 569), «tu li conosci gli inglesi?» (GF: 571) -, "che" polivalente - «come tutte le altre volte che beccano uno della repubblica» (GF: 549) -, "ci" presentativo - «c'è da guadagnarci» (GF: 551), «ci sarà, mettiamo degli inglesi» (GF: 555), «c'è pericolo che si formino il concetto» (GF: 569), «non c'è nessun pericolo» (GF: 570), «c'è il cuciniere che prima o poi» (GF: 571), «c'è Elia con la scabbia» (GF: 571) -, casi di mancato accordo tra soggetto e verbo - «l'ha preso Tarzan e la sua volante» (GF: 550), «ci sarà, mettiamo, degli inglesi» (GF: 556) -, raddoppiamento pronominale - «a lavarci i piatti a noi» (GF: 556 ), «a te ti farò vedere io» (GF: 557) -, pronomi di affetto - «se [...] mi spuntasse da un'altra parte un fascista» (GF: 555), «noi ce lo facciamo soltanto tra noi» (GF: $555)-$.

Sono frequenti i segnali discorsivi - «dico io» (GF: 554), «mettiamo» (GF: 556), «di'»(GF: 556), «guarda» (GF: 555) «mah» (GF: 556) -, e alte espressioni con carattere intensificatore: «com'è vero Dio» (GF: 555), «le balle» (GF: 556), «certo che» 
(GF: 562), «addio» (GF: 570); ricorrono spesso anche i deittici temporali e spaziali - «qui», «quassù», «su», «giù», «appena», «ora», «tutt'oggi», «allora», «subito»-. Tutti questi fenomeni si riscontrano in modo frequente in alcune opere ascritte dalla critica alla narrativa cosiddetta neorealista; ne costituiscono anzi, secondo Enrico Testa (1997: 226), la «vera cifra stilistica», in particolare le frasi segmentate e le frasi scisse. In questo racconto di Fenoglio, come Testa (1997: 231) rileva per i romanzi coevi, è visibile da un lato «un'adozione generalizzata di fenomeni e costrutti parlati entrati ormai a pieno titolo nello standard» e dall'altro il «ricorso a forme la cui "extrasistematicità" coincide col loro spiccato tenore orale».

Se l'esemplificazione dei fenomeni riconducibili al primo caso risulta abbastanza omogenea nella letteratura del primo dopoguerra, è fondamentale ricondurre il parlato di Fritz qui esemplificato alla seconda istanza identificata da Enrico Testa. Si tratta in effetti di fenomeni al di fuori della consuetudine non soltanto letteraria ma anche orale, in quanto rappresentanti di un processo comunicativo difficoltoso portato alle sue estreme conseguenze facendo parlare per buona parte del racconto un tedesco che con estrema difficoltà riesce ad esprimersi in lingua italiana. È tuttavia necessario distinguere il racconto di Fenoglio dalle opere degli altri autori presi in considerazione. La presenza di un parlante tedesco che interagisce con i partigiani e i civili italiani si differenzia sostanzialmente rispetto agli altri testi presi in considerazione.

In Banditi e in Uomini e no è completamente assente il tentativo da parte degli italiani di semplificare la propria lingua al fine di favorire una maggiore comunicazione con i tedeschi. Nel libro di Chiodi (1946 [2015]: v), che si pone l'obiettivo di non essere «un romanzo, né una storia romanzata» ma «un documentario storico», nessun italiano tenta di trovare una lingua semplificata adatta alla comunicazione. I rapporti che si instaurano tra i partigiani, i civili e i tedeschi sono rigidamente inquadrati in una «antitesi di natura manichea» (Corti 1970: 63) perfettamente aderente alla visione schematica e agiografica di certa letteratura resistenziale. Il libro di Chiodi, che Beccaria (2015: vii) definisce un diario «crudamente e oggettivamente realistico», non lascia spazio al dubbio nella rappresentazione delle forze in campo.

Lo stesso accade, in maniera diversa, in Uomini e no. Gli esempi di parlato dei tedeschi che sono stati presi in considerazione fungono esclusivamente da esempi concreti dell'italiano parlato dalle forze di occupazione. L'intento di Chiodi e di Vittorini non è quello di rappresentare il dialogo intercorrente tra italiani e tedeschi, essi intendono rappresentare l'incomunicabilità tra i due diversi mondi rappresentati. Lo stesso avviene nel romanzo di Calvino (1947 [2011: 25]), dove «i tedeschi non capiscono quello che si dice». I dialoghi che intercorrono tra Pin e i tedeschi risultano sempre volti non alla comunicazione ma alla derisione del tedesco, considerato sempre e soltanto un nemico. La comunicazione tra Pin e i tedeschi, che all'inizio del romanzo ancora si presentava come dialogo, in chiusura si semplifica tanto da trasformarsi in semplice onomatopea: «"La pecora" dice "avete mica visto la mia pecora?" "Was?" I tedeschi non capiscono. "Una pecora. Pe-co-ra. Bèeee... Bèeee..."” (Calvino 1947 [2011: 141]).

Il racconto di Venturi pare essere quello che idealmente si avvicina maggiormente linguisticamente a Golia. Il contadino protagonista, infatti, interagisce con il tedesco che stentatamente prova a parlare in italiano nel modo descritto sopra. Si possono osservare alcuni fenomeni di semplificazione linguistica volti a favorire la comunicazione, senza però che essi rappresentino una rivisitazione del rapporto tra i due schieramenti né una messa in discussione dei ruoli ad essi attribuiti. Si tratta 
di una ripetizione sconsolata e quasi ironica dello sgrammaticato italiano parlato dai tedeschi, ancora una volta per sottolinearne l'alterità: "“Io niente capire”, disse il tedesco. "Tu niente capire, il contadino disse. - Ma io lo so cosa voglio dire"» (Pedullà 2005: 308); "“I tuoi camerati, cercò di spiegare. - Quelli della vacca, mi portarono via anche le galline. Ne avevo sette. E mentre io parlavo col tenente, per via della vacca, gli altri presero tutte le galline che erano sull'aia. Quando me ne avvidi corsi dietro al tenente e gli dissi: Gesù benedetto, ma qui come faccio, allora? Che cosa darò da mangiare al mio bambino? Ma il tenente disse: Niente capire"» (Pedullà 2005: 308).

Nel racconto di Fenoglio, come si è visto, la comunicazione tra italiani e tedeschi prende una piega diversa, in quanto non soltanto il tedesco tenta di parlare al meglio una lingua per lui difficile e poco conosciuta ma gli stessi italiani (in un rapporto di forza ribaltato rispetto agli episodi tratti dalle opere degli altri autori) cercano una lingua facilmente accessibile al prigioniero. Si può affermare che l'intento mimetico degli autori presi in considerazione si realizza in modi diversi (non soltanto quantitativamente) rispetto al racconto di Fenoglio, perché diverse sono le intenzioni dei narratori degli anni Quaranta rispetto al racconto fenogliano che, per quanto linguisticamente ancora legato alla tradizione dell'immediato dopoguerra, inizia a mettere in dubbio l'invariabilità di un canone destinato ad essere superato.

Nella prima redazione del Partigiano Johnny compare un unico caso di "dialogo", se così lo si può chiamare, tra i partigiani e i tedeschi. Un incidente stradale permette la cattura di alcuni tedeschi, ma i due mondi appaiono, nella descrizione fornita da Fenoglio, inconciliabili e ogni forma di comunicazione risulta impossibile: «Nulla accadde in quel paio d'ore, i tedeschi non fecero altro che accudire il loro ufficiale, parlando in un tedesco stretto, ma lento e affettuoso, con molta irritazione di René incapente che un paio di volte ineffectualy burst out con un Che dite, maiali tedeschi?» (Fenoglio 1978: 502). Tale schematizzazione dell'impossibilità comunicativa viene in parte superata nella seconda redazione. Al recupero del medesimo episodio (Fenoglio 1978: 931) si aggiunge una situazione differente: "Chi precedeva Johnny nella coda al calderone era un ufficiale di forse quarant'anni, alto e forte e con una faccia straniera. - Capitan Asther, - gli domandò un suo compagno della Prima Divisione, - che cosa ti faranno domani i tuoi fratelli tedeschi? Asther - un tedesco dunque, con una testa massiccia ed un profilo sfuggente - sorrise e con l'aiuto del mestolo mimò molto sommariamente il taglio della gola» (Fenoglio 1978: 1021). Se da un lato si può ancora osservare una comunicazione prettamente fisica, i due mondi opposti entrano finalmente in contatto. Il tedesco, dunque, si presenta in una condizione mutata. L'«incapente» René, emblema di certa schematizzazione agiografica della Resistenza, appare annullato dalla comunicazione, per quanto sommaria, che Asther mette in atto. Il passaggio dalla prima alla seconda redazione del Partigiano, quindi, realizza un processo di messa in discussione, anche se sarebbe forse eccessivo definirlo revisionista, del modello resistenziale del decennio precedente. Su questa impostazione si colloca il racconto qui analizzato, sebbene al livello ancora successivo di vero e proprio ribaltamento di prospettiva.

Molto diversa a livello sintattico si presenta la lingua utilizzata nelle parti narrative del racconto. Una prima differenza è riscontrabile nella lunghezza variabile delle frasi, più estese in corrispondenza delle sezioni strettamente narrative - ad es. «Il drappello puntava per salire, gli altri per fermarlo, sicché a chi guardava dalla spe- 
cola pareva che tutt'insieme si affannassero a spingere su per l'erta un pesantissimo carro che un po' sale ma poi riscivola quando lo sforzo si rompe» (GF: 549-550) - e più brevi in corrispondenza delle parti dialogate - «Così parlò Fritz, con la voce avvampante per il riflesso della stufa. Il discorso aveva affrettato l'ora di togliere il disturbo» (GF: 566) -.

Si può osservare una maggiore frequenza dell'ipotassi comprendente una maggiore varietà di subordinate: proposizioni finali - «Il drappello puntava per salire» (GF: 549) -, consecutive - «Il drappello puntava per salire, gli altri per fermarlo, sicché a chi guardava dalla scuola pareva che tutt'insieme si affannassero a spingere su per l'erta un pesantissimo carro» (GF: 549) -, temporali - «La gente fremette e serrò a gomito a gomito quando su di essa, in curva, passò lo sguardo di lui» (GF: 550) -, causali -«Poi di Fritz [...] Sandor doveva essersi fatto un buon concetto, perché lo mise fuori a spaccar legna» (GF: 552) -, relative - «erano molto più stranieri Tom e Victory, che l'otto settembre erano evasi da un campo di concentramento» (GF: 553) -, comparative - «Il colosso si portò una mano al petto, come se lì fosse stato ferito» (GF: 550) -, concessive - «Benché il tedesco indugiasse dietro la grata, ora la gente non aveva più occhi che per la sua calzatura» (GF: 552) -, oggettive $-«$ Vide Polo andare al portico e tirarne fuori il camion» (GF: 557) -, «Si vide però il più piccolo ed il più giovane dei partigiani $[. .$.$] avvicinarsi più di tutti$ al gigante» (GF: 550), interrogative indirette - «Uno si schiarì la gola e domandò se lo fucilavano» (GF: 551) -.

A livello paratattico si osserva il frequente utilizzo della giustapposizione per asindeto - «Negli stomaci le torte si rapprendevano e pesavano come cemento, dentro il vino correva a gara col sangue, la stufa era incandescente» (GF: 562-563), «Della repubblica non era, tedesca non pareva, ad ogni buon conto lo puntarono con tutte le armi» (GF: 568) -, sebbene spesso le proposizioni coordinate giustapposte siano mescolate liberamente a proposizioni introdotte da una vasta gamma di congiunzioni: copulative («e», «né»), avversative («ma», «invece»), disgiuntive $(« 0 »)$. L'uso della congiunzione «e» è diffuso sia nelle parti dialogate che in quelle narrative e il suo utilizzo in genere, nelle opere di Fenoglio, «risponde a un' istanza di sintesi» (Testa 1997: 319) che pare però risolversi in questo racconto in una serie di elenchi di azioni - «E nel mentre parlava e cantava e gesticolava come uno zingaro, con certe scrollate elettriche» (GF: 566), «Arrivati al comando, ne parlo subito al maggiore e vedrai che lui ti dirà di portarglielo giù e ti farà un cicchetto perché non hai provveduto a suo tempo» (GF: 570$)-$.

Influisce sulla caratterizzazione della lingua delle parti narrative anche la presenza di verbi riflessivi dal senso straniato - «s'erano lottati» (GF: 549), «da potersi» (GF: 559), «Fritz s’avanzò» (GF: 561) -. Anche nelle sezioni narrative sono presenti tratti dell'oralità quali frasi segmentate - «come se non n'avesse parecchie di quelle occasioni» (GF: 549), «le ultime parole le disse» (GF: 564) -, riprese che danno il sapore del racconto orale popolare - «Il terzo giorno, due donne che tornavano dal forno con un bambino per mano e nell'altra il cestone del pane, queste due donne [...]» (GF: 551) -, "che" polivalente - «ci fu un giorno che il tedesco dovette tremare per la sua vita» (GF: 557), «quando gli riusciva un fendente di particolare forza e precisione, che le schegge rimbalzavano [...]» (GF: 552), «dopo aver chiuso i bambini in casa e sbarrato tutto, che non potessero vedere assolutamente niente» (GF: 558), «riempiendo i dormiveglia di fantasie libidinose [...], che una donna per tutta una notte gli pitturasse con lacca azzurra la pianta dei piedi» (GF: 567) -, "ci” pre- 
sentativo - «ci fu un giorno» (GF: 557), «ci fu poi un altro avvenimento» (GF: 560), «c'era da restare accecati» (GF: 571)-.

Sempre in direzione dell'oralità si può osservare la presenza di segnali discorsivi propri dell'oralità («basta», «ora», «chissà», «insomma», «e via»). «Distorsioni grammaticali minime» (Soletti 1987: 62), invece, risultano, secondo Elisabetta Soletti, le alterazioni dovute all'ambiente fortemente dialettale nel quale si muoveva Fenoglio, come ad esempio le doppie preposizioni («di tra le sbarre», «da sulla tavola»). In senso opposto si collocano le costruzioni sintattiche latinizzanti - «Ora il cuciniere lo mandava spesso in giro per le case a farsi imprestare gli arnesi da cucina che a lui mancavano, e dopo avergli dato quello che gli bisognava, le donne lo trattenevano sempre un po' e gli versavano vino dolce, la prima che aveva pensato d'offrirglielo avendo scoperto che gli piaceva più dell'altro ed essendosi fatta premura d'avvisarne le compagne» (GF: 553) -, le subordinate con verbo all'infinito dal sapore arcaizzante - «sembrava essere la frontiera del mondo, da lassù potersi fare il tuffo senza fine» (GF: 571) - e la costruzione arcaizzante di participi passati accordati al complemento oggetto - «s'erano presa la testa» (GF: 565), «s'era accesa una sigaretta» (GF: 569) -.

Secondo Gian Luigi Beccaria (1984: 18) le «costruzioni forzatamente latineggianti» sono volute dall'autore e non dovute «ad impossibilità da parte dell'autore di svolgere l'enunciato in un più bello stile ed in più buona lingua». Infine, pur essendosi Fenoglio liberato dalla «preoccupazione ossessiva in Italia del bello stile e della bella lingua» (Beccaria 1984: 18), si possono notare altri fenomeni propri di uno stile letterario, come ad esempio l'aggettivazione bimembre - «il più piccolo ed il più giovane dei partigiani» (GF: 550), «una nuova e più alta responsabilità» (GF: 550), «occhi trepidi e compiaciuti» (GF: 551) - e trimembre - «una pelle di bimbo [...] tenera, abbondante, fulgida per i peli d'oro» (GF: 552) -. Nella sintassi del racconto convivono dunque «componenti che appartengono a registri diversi»e in tale ibridismo si può scorgere «un gesto intenzionale di rottura della media linguistica attribuibile a un clima letterario posteriore al neorealismo e ad esso relativo» (Grignani 1978: 317).

La punteggiatura non risulta avere un ruolo differenziato a seconda che si trovi nelle parti dialogate o in quelle narrative. Da notare a questo proposito è l'utilizzo, nella quasi totalità dei casi, della virgola insieme alla congiunzione «e» per introdurre le proposizioni coordinate. Ad esempio, per le parti narrative: «S'erano lottati così per un quarto d'ora, e il groviglio sbandava $[\ldots]$ (..] (GF: 549), «portava sul braccio un fagottone bianco, e dietro gli uscì la moglie del dottore» (GF: 557); per le parti dialogate: «[...] si deve far del male a tutti, e noi ce lo facciamo soltanto tra di noi» (GF: 555), «Ma se invece ci pigliamo tra noi, niente ti salva più, e se cerchiamo di spiegare $[\ldots] \gg(\mathrm{GF}: 556)$.

Veniamo ora al lessico, iniziando dalle caratteristiche che riguardano la presenza di un parlante tedesco tra parlanti italiani. Un elemento fondamentale è rappresentato dalle parole tedesche che sporadicamente vengono pronunciate da Fritz: «Sie» (GF: 563), «Kartoffel» (GF: 563), «Englisch!? Englische streitenuniform» (GF: 570). Il lessico italiano che utilizza il prigioniero nel corso di tutto il racconto è estremamente ridotto e comune. Le parole utilizzate sono di uso comune e continuamente ripetute, senza l'ausilio di sinonimi: «buono era Tarzan» (GF: 560), «tante altre cose buone per festa» (GF: 562), «buono fuoco» (GF: 565), «buono soldato tedesco» (GF: 573), «buono partigiano» (GF: 573), «belli bambini» (GF: 551), «capace» (GF: 567), «ca- 
pace fare affari» (GF: 571), «capace di uccidere me» (GF: 574), ecc) con la presenza dell'unico neologismo «canze» (GF: 562) per 'canzoni'. Il lessico utilizzato nelle parti dialogate da partigiani e abitanti del paese risente in parte della presenza di Fritz, rivolgendosi al quale vengono utilizzate parole tedesche.

Il «mezzo tedesco» del signor Ilario rende evidente un processo di semplificazione linguistica paragonabile a quello del prigioniero tedesco: «Ich kriegsgefangen in Osterreich» 'io prigioniero di guerra in Austria' (GF: 563), «Mangiare sempre kartoffel» 'patate' (GF: 563), «Papier, sempre vestiti di papier» 'carta' (GF: 563), «im winter und arbeit in bahnen» 'in inverno e al lavoro in strada' (GF: 563), «nicht brot, nicht feuer» 'non pane, non fuoco' (GF: 564). Il tedesco minimo utilizzato da Carnera per comunicare con Fritz, invece, pare all'opposto la rappresentazione di un processo comunicativo difficile nel quale il soggetto in posizione di forza tende a ridurre al minimo l'abbassamento del proprio livello linguistico limitandolo a singole parole fondamentali per la trasmissione di un concetto: «Andiamo a fare un giro intorno al paese. Spazziren, eh?» (GF: 571) storpiatura di spazieren 'passeggiare' e «Guarda, Fritz, che ti faccio kaputt» (GF: 574)).

Anche in Chiodi e Vittorini sono riscontrabili esempi di inserimento di vocaboli tedeschi all'interno del testo. Le differenze con il testo di Fenoglio risultano però evidenti. In Chiodi le frasi e le parole in tedesco - «C'è il Lagerfürer con un'impiegata» 'comandante di campo' (Chiodi 1946 [2015: 79]), "Tre parole sono sempre sulla bocca dei vecchi internati: Krenkeit, Polizei, Reichenau»'malattia, polizia, Reichenau' (Chiodi 1946 [2015: 82]), «Aufwiedersen, Herr Professor» 'arrivederci, signor professore' (Chiodi 1946 [2015: 85]), «Il signore che mi accompagna mi raccomanda di portare quel foglio all'Arbeitsamt» 'commissione del lavoro' (Chiodi 1946 [2015: 85]) - sono utilizzate nelle parti narrative e dialogate riferite alla prigionia dell'autore / protagonista in Austria. Sembrano dunque avere esclusivamente una funzione mimetica e rappresentativa di una condizione nella quale l'autore si è ritrovato. Anche in questo caso viene meno la rappresentazione di una lingua mescidata con fine comunicativo. Rilevante a questo proposito è il fatto che il tedesco presente in Banditi si trova sempre ed esclusivamente su cartelli e scritte in Austria o nella bocca dei tedeschi. Anche sul piano lessicale manca il tentativo di avvicinamento linguistico riscontrato in Fenoglio, dove, per l'appunto, il tedesco ricorre anche nelle frasi degli italiani.

In Uomini e no la presenza di frasi e parole in tedesco è molto consistente: «Drei kleine Knochen» (Vittorini 1945 [1981: 53]), «Sag ihnen dass es kein Questore gibt... Und frage von welcher seite es weggefahren ist» (Vittorini 1945 [1981: 65]), «Dann weiter!», «Wer ist's gewesen?» (Vittorini 1945 [1981: 105]), ecc. Anche in quest'opera tutti gli esempi di tedesco riscontrabili sono da ricondurre a parlanti tedeschi, ad eccezione del tentativo di un ufficiale tedesco di insegnare a un soldato italiano a contare fino a cinque (Vittorini 1945 [1981: 67-69]). In entrambe queste opere manca dunque, come si è già detto, la rappresentazione di una sorta di lingua franca comprensibile da italiani e tedeschi e costituita, oltre che dalla semplificazione sintattica analizzata in precedenza, anche dall'inserimento di lessico tedesco.

La presenza del tedesco nel racconto di Fenoglio non deve essere considerata un esempio di volontà espressionistica, ma al contrario tale fenomeno potrebbe essere inteso in prima istanza come mimesis di una interlingua generata dall'incontro fra italiani e tedeschi. Oltre all'intento mimetico, però, il fenomeno, assente negli altri 
autori, sembrerebbe svolgere una funzione di secondo livello, cioè quella di mettere in discussione le opposizioni nette tra partigiani e tedeschi. Si possono trovare nelle parti dialogate anche esempi di parolacce e bestemmie - «le balle» (GF: 556), «Dio Cristo» (GF: 555), «Dio santo» (GF: 570) -, popolare - «acchiappo» (GF: 555), «pigliamo» (GF: 555) -, di modi di dire - «vengono nelle mani» (GF: 554), «faccio la pelle» (GF: 555), «ci lasciano la pelle» (GF: 561), «l'ha portato al sentimento» (GF: 564), «prendo di qua» (GF: 568), «è una pasta frolla» (GF: 570), «stessero secchi» (GF: 571) - e di lessico marcato regionalmente: «scugnizzo» (GF: 569), «filoni» (GF: 570), «cicchetto» (GF: 570). Sono presenti anche alterati dispregiativi:«bastardone» (GF: 573), «tedescaccio» (GF: 566).

Anche le parti narrative del racconto presentano caratteristiche particolari dal punto di vista lessicale. Sono presenti vari livelli di lessico, molto differenti tra di loro. Si possono notare gli esempi di lessico quotidiano - «dottrina» (GF: 553) nel senso di 'catechismo', «flanella» (GF: 552), «in folle» (GF: 558), «medico condotto» (GF: 557), «sudor freddo» (GF: 559), «toppa» (GF: 552) -, popolare - «beccamorto» (GF: 571), «ghiaccione» (GF: 566), «pigliamo» (GF: 555), «vecchi» (GF: 560) nel senso di 'genitori'-, regionale o dialettale, che svolge una funzione limitata nella scrittura di Fenoglio dove ha lo scopo di «rinvigorire la lingua letteraria» (Beccaria 1984: 43): «abbrivarlo» (GF: 557), «bricco» (GF: 572), «mica» (GF: 551), «rittani» (GF: 559), «zolfini» (GF: 560). I dialettismi si fondano su una forma italiana settoriale-gergale: ad esempio il dialettismo «abbrivare», presente in questo racconto e derivato dal piemontese «brivè» ma retto dalla forma italiana del lessico marinaresco «abbrivare» (Beccaria 1984: 43) o su una matrice dialettale generica (Beccaria 1984: 44). Lo spazio ristretto (e risolto spesso con un lessico non reale ma ipotetico) concesso al dialetto e al linguaggio popolare dimostra che Fenoglio «non abbassava la lingua attraverso la contaminazione col dialetto come lingua bassa, macchia diminutiva o mimetica, realtà geografica socialmente determinata. Non si tratta di calata verso il basso o, altra volta, di ascesa verso l'alto» (Beccaria 1984: 46). Sono presenti anche modi di dire: «appostati in mira» (GF: 551), «a fil di logica» (GF: 557), «ad ogni buon conto» (GF: 568), «cosa di chiesa» (GF: 558), «facevano la pietà» (GF: 559), «tanta pratica di uomini» (GF: 564).

Valgono dunque anche per il lessico di questo racconto le affermazioni di Gino Rizzo in riferimento all'incompiuto Frammenti di romanzo. Rizzo rileva una «evidente predilezione per una lingua usuale con frequenti passaggi colloquiali derivati dal parlare comune» (Rizzo 1976: 47). Sono inoltre presenti esempi di lessico tecnico militare - «Colt 45» (GF: 569), «culatta» (GF: 568), «drappello» (GF: 549), «fondina» (GF: 568), «inquadrato» (GF: 569), «mitraglia» (GF: 559), «pistolino 6,35» (GF: 552), «raffica» (GF: 559), «sentinella» (GF: 549), «servizio di guardia» (GF: 552), «uniforme» (GF: 549) - e resistenziale ${ }^{6}$ - «comando» (GF: 568), «cuciniere» (GF: 553), «rastrellamenti» (GF: 569), «repubblica» (GF: 559), «sbandamento» (GF: 553) -, aulico e letterario - «erta» (GF: 549), «barbaglio» (GF: 573), «lizza» (GF: 567), «schifiltosaggine» (GF: 568), «abbrivo» (GF: 574), «memento» (GF: 552), «s'intorbidavano» (GF: 552), «impietrati» (GF: 560), «illanguiditi» (GF: 560) -. Sono presenti anche rari casi di arcaismi: il locativo «vi» (GF: 566), «debbono» (GF: 554). Gli esempi di lingua letteraria e di arcaismi sono in numero

Maria Corti (1978: 92-93) considera tali termini tecnici come una componente rilevante della narrativa resistenziale. 
ridotto e limitati alle parti narrative del racconto, secondo lo «stile alto» che Gian Luigi Beccaria identifica con l' «elevare la lingua attraverso riecheggiamenti letterari di antico» (Beccaria 1984: 50). L'esemplificazione qui riportata colloca il racconto analizzato in continuità con l'opera di Fenoglio, condensando e rendendo visibili in poche pagine i principali fenomeni lessicali studiati nelle sue opere.

La formazione delle parole non presenta sostanziali differenze tra le parti dialogate e quelle narrative. Una particolare ricorrenza è quella degli aggettivi e degli avverbi al grado superlativo («biondissimi», «pesantissimo», «stanchissimo», «lentissimamente»). Sempre Beccaria (1984: 19) rileva che «la novità di Fenoglio scrittore sta nell'usare una lingua caricata, in tensione, di grado forte e superlativo». Da notare anche la prefissazione (Beccaria 1984: 56-57) utilizzata per le forme verbali: «strabevuto» (GF: 565), «diseguagliata» (GF: 571).

\section{Conclusioni}

Racconto corale, uso del discorso indiretto libero, piena aderenza al livello colloquiale / popolare della lingua coincidono con gran parte dello stile narrativo di adesione alla realtà che anima il dopoguerra e risultano in sintonia con l'intento mimetico funzionale a esprimere l'esperienza bellica e della Resistenza diffuso in quegli anni. Qui Fenoglio però va oltre: nella rappresentazione della lingua del tedesco e dei partigiani si spinge a un livello di mimesi non sperimentato da altri prima. La funzione di questo straordinario esperimento non è solo quello di sviluppare in maniera implicita il concetto di interlingua ma soprattutto quello di utilizzare la rappresentazione di una situazione estrema per mettere in discussione i canoni della letteratura resistenziale. Il ruolo di protagonista attribuito a un tedesco e quello di antagonista a un partigiano e il ribaltamento della prospettiva biblica indicata dal titolo contribuiscono a fornire un'inedita rappresentazione dei rapporti di forza tra gli schieramenti impegnati nella guerra. Se forse parlare di revisionismo è eccessivo, non si può fare a meno di notare che tale racconto dovrebbe essere collocato nella stessa prospettiva di messa in discussione della rigida contrapposizione tra tedeschi e partigiani propria della seconda redazione del Partigiano Johnny ${ }^{7}$ e di Una questione privata.

L'analisi linguistica effettuata ha tentato, dunque, di evidenziare il fatto che questo racconto pur presentandosi come in linea con la tendenza alla mimesis propria del dopoguerra, presenta numerosi elementi di allontanamento dal "canone" della letteratura resistenziale. Alberto Casadei (2004: 142) considera il Partigiano Johnny «lo snodo fondamentale del percorso letterario fenogliano», sottolineandone il distacco «dai principali modelli di narrativa resistenziale, e soprattutto dalla memorialistica più o meno ravvicinata all'evento». Per una più completa analisi dell'allontanamento di Fenoglio pare necessario confrontarsi anche con questo racconto, che, sebbene sia lontano dallo stile epico del Partigiano, rappresenta un momento certamente fondamentale della narrativa fenogliana.

La narrativa resistenziale vede la sua nascita, secondo Maria Corti (1978: 34), nella tradizione orale contemporanea alla Resistenza. Al momento successivo, quello della memorialistica immediatamente successiva all'esperienza bellica, prende

Contribuisce ad avvicinare il racconto a questa redazione anche l'esiguità di tratti marcatamente espressionistici. 
parte Beppe Fenoglio con gli Appunti partigiani, pubblicati soltanto nel 1994. Questo scritto risulta «in bilico tra memorialistica e narrativa breve» (Grignani 1998: 21) e presenta molte delle caratteristiche che sono state analizzate anche nel racconto qui preso in considerazione, riferibili alla narrativa partigiana della prima ora. $\mathrm{E}$ necessario prendere in considerazione questo punto di partenza, oltre a quello che rappresentano Il partigiano Johnny e Una questione privata, per prendere atto del passaggio fondamentale che il racconto Golia rappresenta nel distacco dalla narrativa impregnata di «ottimismo esistenziale-ideologico» (Corti 1978: 36) degli anni immediatamente precedenti.

Come è stato illustrato, questo racconto presenta una forte istanza mimetica, volta a rappresentare un italiano semplice e trasformato dalla scarsa conoscenza linguistica di un tedesco. Ricorrono inoltre tutti gli elementi linguistici propri della narrativa fenogliana, volti però non alla minuziosa ricostruzione degli avvenimenti della guerra civile ma alla riflessione sul significato stesso della guerra. La peculiarità del racconto, cioè la presenza di un tedesco con una scarsa conoscenza della lingua italiana, è volta non al solo intento mimetico. La comunicazione che intercorre tra Fritz e i partigiani rappresenta un esempio di semplificazione linguistica allo scopo di favorire la reciproca comprensione. A differenza delle altre opere prese in considerazione, dove la presenza della lingua tedesca e degli stessi parlanti tedeschi era mirata a rappresentare anche sul piano linguistico la distanza tra due mondi inconciliabili posti agli opposti di un'antitesi irrisolvibile (Corti 1978: 63), il racconto di Fenoglio si allontana dal punto di vista «unico, cui si contrappongono i "non punti di vista"» (Corti 1978: 71).

La mimesi linguistica, così fondamentale nella letteratura resistenziale, viene utilizzata da Fenoglio nella sua più estrema esemplificazione, arrivando a ipotizzare una lingua di comunicazione fruibile da tedeschi e italiani, ribaltando però la stessa prospettiva sulla Resistenza. La stessa possibilità di uno scambio dialogico tra un tedesco e i partigiani pone il racconto al di fuori dell'immaginario degli scrittori (e del pubblico) abituati a una prospettiva fortemente modellata su rigide contrapposizioni, non in grado di «dare una visione non parziale e deformata della realtà» (Chicco Vitizzai 1977: 76). Il ribaltamento di prospettiva, con un tedesco protagonista del racconto e un partigiano italiano come antagonista che lo uccide immotivatamente, rappresenta uno snodo fondamentale per il passaggio alla rappresentazione della guerra civile che Fenoglio realizzerà in Una questione privata e Il partigiano Johnny.

La crisi personale di Fenoglio del 1954, che seguì il risvolto di copertina della Malora scritto da Vittorini, testimonia «le sue personali difficoltà a trovare una strada narrativa che superasse i rischi del neoverismo senza tornare allo "scrivere bene" passatista» (Casadei 2013). Pur nell'incertezza sulla data di composizione del racconto, si può considerare questo testo come fondamentale per cogliere la crisi della letteratura memorialistica e apologetica dell'esperienza partigiana e il contemporaneo sviluppo della narrativa fenogliana. Una lingua ancora lontana da quella che sarà usata nel Partigiano, ma che, nonostante questa lontananza sia dalla Malora sia dagli Appunti partigiani, si colloca nella fondamentale fase di passaggio tra una fase forse più vicina alla tradizionale letteratura resistenziale della scrittura di Fenoglio e una nuova fase di «visione e comprensione superiore degli eventi» (Casadei 2004: 163), nella quale, però, non viene messo in dubbio il valore etico della Resistenza. In questo contesto si genera «il ripensamento che porterà Fenoglio a scrivere la sua esperienza di partigiano [...] così come la rileggeva a distanza di un decennio e più» 
(Casadei 2013), avendo dunque avuto il tempo non solo di riflettere sul significato esistenziale della scelta partigiana, ma sulla guerra civile nel suo insieme. Fenoglio mette in dubbio una visione della realtà troppo ideologizzata e priva della necessaria «oggettivizzazione del vissuto» (Casadei 2004: 198), con l'intenzione di dimostrare che, sebbene la ragione sia indiscutibilmente dalla parte dei partigiani, ciò «non comporta però necessariamente che il bene e il male, gli orrori della guerra, le inutili violenze, la pietà e l'umanità siano distribuiti secondo criteri politici e nazionali»» (Guthmüller 2011: 155).

\section{Riferimenti bibliografici}

Alfano, Giancarlo (2014): «L'irredimibile circuito dell'origine», in Gabriele Pedullà (a c. di), Beppe Fenoglio, L'illuminista, anno XIV, nn. 40/41/42, , pp. 739-761.

Baldini, Anna (2013): «Il Neorealismo. Nascita e usi di una categoria letteraria», in Irene Fantappiè, Michele Sisto (a c. di), Letteratura italiana e tedesca 1945-1970: campi, polisistemi, transfer / Deutsche und italienische Literatur 1945-1970: Felder, Polysysteme, Transfer, Roma, Istituto italiano di Studi germanici, pp. 109-128.

Beccaria, Gian Luigi (1984): La guerra e gli asfodeli, Milano, Serra e Riva.

Berruto, Gaetano (2003): Fondamenti di sociolinguistica, Roma/Bari, Laterza.

Bigazzi, Roberto (1983): Fenoglio: personaggi e narratori, Roma, Salerno editrice.

Bufano, Luca (1999): Beppe Fenoglio e il racconto breve, Ravenna, Longo editore.

Calvino, Italo (1947 [2011]): Il sentiero dei nidi di ragno, Milano, Mondadori.

Calvino, Italo (1947 [2011]): «Prefazione», in Italo Calvino, Il sentiero dei nidi di ragno, Milano, Mondadori, pp. v-xxv.

Casadei, Alberto (2013): «Fenoglio oggi», Le parole e le cose (12 dicembre), <www.leparoleelecose.it>.

Casadei, Alberto (2004): Stile e tradizione del romanzo italiano contemporaneo, Bologna, il Mulino.

Chicco Vitizzai, Elisabetta (a c. di) (1977): Il neorealismo: antifascismo e popolo nella letteratura dagli anni trenta agli anni cinquanta, Torino, Paravia.

Chiodi, Pietro (1946 [2015]): Banditi, Torino, Einaudi.

Corti, Maria (1980): Beppe Fenoglio. Storia di un continuum narrativo, Padova, Liviana Editrice.

Corti, Maria (1978): «Neorealismo», in Maria Corti, Il viaggio testuale, Torino, Einaudi, pp. 25-110.

Corti, Maria (1970): «Realtà e progetto dello scrittore nel fondo Fenoglio», Strumenti critici, 11, pp. 38-59.

De Nicola, Francesco (1996): Neorealismo, Milano, Editrice Bibliografica.

Fenoglio, Beppe (1978): Opere, a cura di Maria Corti, Torino, Einaudi, 3 voll., 5 tomi.

Fenoglio, Beppe (1992): Romanzi e racconti, a cura di Dante Isella, Torino, Einaudi.

Fenoglio, Beppe (2007): Tutti i racconti, a cura di Luca Bufano, Torino, Einaudi.

Grignani, Maria Antonietta (1998): «Appunti sugli "Appunti partigiani», in Pino Menzio (a c. di), Beppe Fenoglio 1922-1997. Atti del convegno. Alba 15 marzo 1997, Milano, Electa, pp. 19-26.

Grignani, Maria Antonietta (1981): Beppe Fenoglio, Firenze, Le Monnier.

Grignani, Maria Antonietta (1978): «Virtualità del testo e ricerca della lingua da una stesura all'altra del Partigiano Johnny», Strumenti critici, 36-37, pp 275-331. 
Guthmüller, Bodo (2011): «Il racconto "Golia” di Beppe Fenoglio», in Tiziana Piras (a c. di), Gli scrittori italiani e la Bibbia. Atti del convegno di Portogruaro, 21-22 ottobre 2009, Trieste, Edizioni dell'Università di Trieste, pp. 145-155.

Luperini, Romano (2001): «Riflettendo sulle date: alcuni appunti sul neorealismo in letteratura», Allegoria, anno XIII, n. 37, pp. 125-132.

Matt, Luigi (2011): La narrativa del Novecento, Bologna, il Mulino.

Milanini, Bruno (a c. di) (1980): Neorealismo. Poetiche e polemiche, Milano, Il Saggiatore.

Rizzo, Gino (1976): Su Fenoglio tra filologia e critica, Lecce, Edizioni Milella.

Selinker, Larry (1972): «Interlanguage. Product Information» International Review of Applied Linguistics in Language Teaching, 10, pp. 209-241.,

Sipione, Marialuigia (2011): Beppe Fenoglio e la Bibbia. Il «culto rigoroso della libertà», Firenze, Franco Cesati.

Soletti, Elisabetta (1987): Beppe Fenoglio, Milano, Mursia.

Soletti, Elisabetta (1984): «La scrittura in bianco e nero», in Gino Rizzo (a c. di), Fenoglio a Lecce. Atti dell'Incontro di studio su Beppe Fenoglio. Lecce, 25-26 novembre 1983, Firenze, Olschki, pp. 155-166.

Testa, Enrico (1997): Lo stile semplice. Discorso e romanzo, Torino, Einaudi.

Thelander. M. (1988): «Analysis of Coexistent Systems», in Ulrich Ammon, Norbert Dittmar, Klaus J. Mattheier (a c. di), Sociolinguistics / Soziolinguistik, Berlin/New York, de Gruyter, pp. 1007-1014.

Venturi, Marcello (1945 [2005]): «Estate che mai dimenticheremo», in Gabriele Pedullà (a c. di), Racconti della Resistenza, Torino, Einaudi, pp. 305-311.

Vittorini, Elio (1945 [1981]): Uomini e no, Milano, Mondadori. 